\title{
CARACTERIZACIÓN NEUROUROLÓGICA DEL MIELOMENINGOCELE EN EL PACIENTE ADULTO.
}

\author{
Fernando Verdú Tartajo, Jesús Salinas Casado', Felipe Herranz Amo, José María Adot Zurbano² \\ y Ramiro Cabello Benavente.
}

Unidad de Urodinámica. Servicio de Urología. Hospital Universitario Gregorio Marañón. Madrid.

Unidad de Urodinámica'. Servicio de Urología. Hospital Clínico Universitario San Carlos. Madrid.

Unidad de Urodinámica². Servicio de Urología. Hospital General Yagüe. Burgos. España.

\begin{abstract}
Resumen.- OBJETIVOS: Caracterizar desde el punto de vista neurourológico, la disfunción vesicouretral que presentan los pacientes nacidos con mielomeningocele (MMC), cuando alcanzan la edad adulta.

MÉTODOS: Presentamos los resultados de la exploración física neurourológica y del estudio videourodinámico de 52 pacientes adultos nacidos con MMC, de edades comprendidas entre los 18 y 40 años (media de 20.7), controlados durante los últimos 14 años en la Unidad de Urodinámica del Hospital General Universitario "Gregorio Marañón" de Madrid. En todos ellos se determinó también el nivel neurológico de su lesión. Según estos resultados, establecemos el diagnóstico de la lesión de los tres elementos constitutivos de la inervación vesicouretral (simpático, parasimpático y pudendo), para posteriormente clasificar la disfunción neurógena del tracto urinario inferior de los pacientes, en base al nivel de la lesión y al tipo de inervación lesionada.
\end{abstract}

Fernando Verdú Tartajo C/ Sangenjo 19, 3 $\mathrm{lzq}$ 28034 Madrid (España) ferverdu@pulso.com Trabajo recibido: 19 de diciembre 2005
RESULTADOS: La alteración en la sensibilidad perineal y la disminución del tono del esfínter anal, junto con la ausencia del control voluntario anal y del reflejo but bocavernoso, fueron los hallazgos más frecuentes en la exploración neurourológica. El comportamiento del detrusor fue predominantemente arrefléxico (88.4\%), asociándose a una acomodación vesical disminuida en la mitad de los casos. El sistema esfinteriano fue incompetente durante la fase de llenado en el $51.9 \%$, siendo competente en el $42.3 \%$ y normal en el $5.7 \%$ restante. Durante la fase de vaciado comprobamos la ausencia de relajación del mismo en el 82.6\%. Existió un predominio del nivel inferior, tanto en la lesión simpática como en la parasimpática y pudenda. En casi todos los pacientes (96.7\%) existía lesión en al menos dos de estos tres tipos de inervación. La disfunción neurógena del tracto urinario inferior más frecuente fue la lesión múltiple pura tipo neurona motora inferior (65.3\%), encontrándose también lesiones puras tipo neurona motora superior (3.8\%), lesiones múltiples mixtas (9.6\%) y lesión única pura (1.9\%). Tres pacientes presentaron una función vesicouretral normal.

CONCLUSIONES: Demostramos la multiplicidad de la disfunción neurógena del tracto urinario inferior en los pacientes adultos nacidos con MMC, la ocasional ausencia de la misma, su falta de correlación con el nivel neurológico de la lesión, y el predominio en éstos de la lesión múltiple pura tipo neurona motora inferior.

Palabras clave: Mielomeningocele. Adulto. Disfunción vesicouretral. Estudio urodinámico.

Summary.- OBJECTIVES: To characterize the neurourological features of the vesicourethral dysfunction of patients with cogenital myelomeningocele when they reach the adult age. 
METHODS: We report the results of the neurourological physical examination and video urodynamic tests of 52 adult patients born with myelomeningocele, with ages ranging from 18 to 14 years (mean age 20.7) controlled over the last 14 years in the Urodynamics Unit of the Hospital Gregorio Marañon in Madrid. The neurological level of the lesion was evaluated in all of them. Following these results we established the diagnosis of the lesion of the three constitutive elements of the vesicourethral innervation (sympathetic, parasympathetic, and pudendal) in order to classify the lower urinary tract neurogenic dysfunction of the patients based on lesion level and type of damaged innervation.

RESULTS: The alteration of the perineal sensitivity and diminishment of the anal sphincter tone, in addition to absence of anal voluntary controlled and bulbocavernosus reflex, where the most frequent findings in the neurourological physical examination. Detrusor behaviour was predominantly areflexic $(88.4 \%)$, in association with diminished bladder compliance in half of the cases. The sphincter was not competent during the filling phase in $51.9 \%$ of the cases, competent in 42.3\%, and normal in the remainder 5.7\%. During the voiding phase we confirmed the absence of sphincter relaxation in $82.6 \%$ of the cases. There was a predominance of the lower level of lesion in all three sympathetic, parasympathetic and pudendal nerves. In almost all patients (96.7\%) there was lesion in at least two of the three types of innervation. The most frequent neurogenic dysfunction of the lower urinary tract was the multiple inferior motor neuron pure lesion (65.3\%), also finding pure superior motor neuron (3.8\%), mixed multiple (9.6\%), and pure single lesions (1.9\%). Three patients presented a normal vesicourethral function.

CONCLUSIONS: We demonstrated the multiplicity of lower urinary tract neurogenic dysfunction in adult patients with congenital myelomeningocele, its occasional absence, the lack of correlation with the neurological level of the lesion, and the predominance of the multiple inferior motor neuron pure type of lesion.

Keywords: Myelomeningocele. Adult. Vesicourethral dysfunction. Urodynamic study.

\section{INTRODUCCIÓN}

Las malformaciones congénitas del tubo neural, abarcan un amplio espectro de anomalías, originadas por una alteración en el desarrollo de las estructuras nerviosas, junto con otras mesenquimales y cutáneas adyacentes.
Entre estas malformaciones se encuentra el Mielomeningocele ( $M M C)$, caracterizado por la falta de fusión de los arcos vertebrales posteriores, asociado a la extrusión de la médula y raíces nerviosas.

Aunque el defecto puede aparecer a cualquier nivel de la columna, muestra predilección por el segmento lumbosacro, de forma que alrededor de un $5 \%$ de los mismos se localizan a nivel torácico, un $26 \%$ en la columna lumbar, un $20 \%$ a nivel sacro y un $47 \%$ lumbosacro, siendo la localización cervical excepcional (1). La afectación neurológica provocada por el $M M C$, varía extraordinariamente, y depende fundamentalmente de los elementos nerviosos contenidos en su saco meníngeo.

El MMC destaca por su frecuente repercusión funcional sobre los aparatos digestivo, locomotor $y$ urinario.

La repercusión funcional sobre el aparato urinario, se manifiesta mediante una alteración de la función vesicouretral, cuyas características han sido claramente establecidas, hasta el inicio de la edad adulta (1-8).

- Presenta una gran variabilidad lesional, pudiéndose encontrar distintos tipos de disfunciones, dependiendo de los elementos nerviosos contenidos en el saco meníngeo, al poder estar afectados uno o más de los 3 elementos constitutivos de la inervación vesicouretral (simpático, parasimpático y pudendo).

- La disfunción no se correlaciona con el nivel neurológico de la lesión.

- Existe la posibilidad de que esta disfunción cambie con el paso del tiempo modificándose durante la infancia o la adolescencia.

- Constituye la principal causa de morbilidad y mortalidad de estos pacientes, superados los dos primeros años de vida.

El hecho de que todavía no haya un gran número de adultos, entre los pacientes nacidos con mielomeningocele, justifica la poca bibliografía existente con respecto a la problemática de los mismos durante esta etapa de la vida.

Los trabajos sobre la situación urológica de estos pacientes son aún más escasos, posiblemente por la pérdida de seguimiento de muchos de ellos al hacerse mayores, y por el hecho de considerar su situación urológica como establecida. 
Con objeto de contribuir al conocimiento de la situación neurourológica de los pacientes nacidos con $M M C$ que han alcanzado la edad adulta, presentamos los resultados obtenidos en una serie de pacientes, que han superado la mayoría de edad.

\section{MATERIAL Y MÉTODOS}

Hemos realizado el estudio neurourológico de 52 pacientes adultos nacidos con MMC, atendidos en el Hospital General Gregorio Marañón durante los últimos 14 años, de los cuales 24 son mujeres y 28 varones.

La edad de los pacientes, en el momento del inicio de su estudio, osciló entre los 18 y los 40 años (media de 20.7) y el tiempo de seguimiento de los mismos entre 1 y 14 años, con una media de 6.7. El defecto óseo de la malformación, en la mayoría de los casos interesaba a las últimas vértebras lumbares y a casi la totalidad de las sacras. Las excepciones fueron 2 casos, con defectos limitados a su columna lumbar, y otro en el que el defecto lumbosacro se extendía a las vértebras dorsales.

Durante la infancia de estos pacientes, en 3 de ellos se había realizado una ampliación vesical con sigma detubulizado, y en otros 4 se habían realizado incisiones endoscópicas del cuello vesical. También durante la infancia, 12 referían el implante de un esfínter artificial AMS 800, de los cuales en 3 la prótesis había sido completamente retirada, en 2 se mantenía únicamente su manguito, y de los 7 pacientes restantes con la prótesis completa, en 4 de ellos funcionaba adecuadamente.

En todos los pacientes se exploró el nivel neurológico de su lesión, entendiendo como tal el segmento más caudal de la médula espinal con función normal en ambos hemicuerpos, y éste se estableció sobre la base de los resultados de la exploración de la sensibilidad, dada la perdurabilidad del mismo, a diferencia del nivel motor, influenciable con el desuso, las contracturas y posibles cirugías. Realizamos la misma división que Schurtleff (9) propuso, basándose en el nivel funcional motor, para el estudio de la problemática derivada de la supervivencia a largo plazo y de la integración social de los pacientes con mielomeningocele, de forma que establecimos tres grupos:

1) Nivel I: Lesión en o por encima de $L_{2}$.

2) Nivel II: Lesión entre $L_{3}-L_{5}$.

3) Nivel III: Lesión en o por debajo de $S_{1}$.

La exploración neurourológica, incluyó la valoración del tono del esfínter anal, así como del control voluntario sobre el mismo, junto con la exploración de la sensibilidad perineal y del reflejo bulbocavernoso. Pudimos recoger los datos relativos a la sensibilidad perineal, el tono anal y el control voluntario del esfínter anal, en 41 pacientes. También,

\section{TABLA I. CRITERIOS DIAGNÓSTICOS DE LA LESIÓN DE LOS COMPONENTES DE LA INERVACIÓN VESICOURETRAL.}

\begin{tabular}{|c|c|c|c|}
\hline LESIÓN & SIMPÁTICA & PARASIMPÁTICA & PUDENDA \\
\hline $\begin{array}{c}\text { TIPO NEURONA } \\
\text { MOTORA SUPERIOR }\end{array}$ & $\begin{array}{c}\text { Disinergia } \\
\text { detrusor-cuello vesical }\end{array}$ & Hiperreflexia & $\begin{array}{c}\text { Disinergia vesicoesfinteriana } \\
\text { y/o } \\
\text { control volutrario anal }(-) \\
\text { tono anal }(+) \\
\text { bulbocavernoso }(+) \\
\text { sensibilidad perineal }(+)\end{array}$ \\
\hline TIPO NEURONA & $\begin{array}{c}\text { II: cuello vesical abierto } \\
\text { MOTORA INFERIOR }\end{array}$ & $\begin{array}{c}\text { disminuida } \\
\text { III: cuello vesical abierto } \\
\text { y acomodación vesical } \\
\text { disminuida }\end{array}$ & $\begin{array}{c}\text { Arreflexia } \\
\text { control voluntario } \\
\text { anal }(-) \\
\text { tono anal (-) } \\
\text { bulbocavernoso (-) } \\
\text { sensibilidad perineal }(-)\end{array}$ \\
\hline
\end{tabular}


en 35 casos, se exploró la presencia o ausencia del reflejo bulbocavernoso.

En todos los pacientes se realizaron estudios videourodinámicos, en los que se registraron simultáneamente la presión vesical y abdominal, obteniéndose la del detrusor por la sustracción de las mismas, junto con el registro electromiográfico de la actividad de la musculatura perineal mediante electrodos de contacto, y la visualización radiológica simultanea del aparato urinario inferior. Para la interpretación de los resultados seguimos las normativas de la I.C.S. (10).

En base a los resultados obtenidos de la exploración neurourológica y de los estudios videourodinámicos, hemos establecido el diagnóstico de la lesión de los distintos componentes de la inervación vesicouretral de acuerdo con los siguientes criterios (1 1) (Tabla I):

1) La lesión del simpático superior viene caracterizada por la presencia de disinergia detrusor-cuello vesical. En la lesión del simpático inferior hemos distinguido tres tipos: Tipo I, definida por la presencia de un cuello vesical abierto durante el llenado; Tipo II, cuando existe disminución de la acomodación vesical y Tipo III, cuando se encuentran las dos alteraciones anteriores.

2) La hiperreflexia vesical (hiperactividad del detrusor neurogénica) manifiesta la lesión del parasimpático superior, mientras que la arreflexia caracteriza la lesión del parasimpático inferior.

\section{NIVEL NEUROLÓGICO}

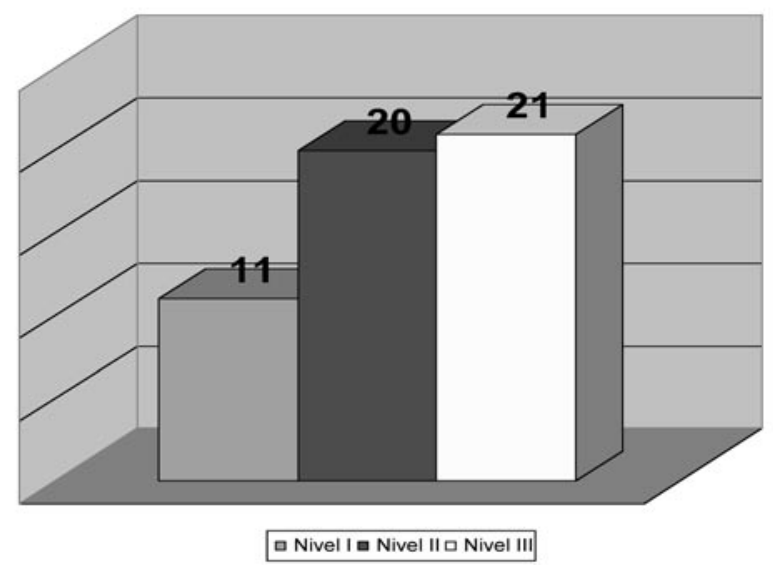

FIGURA 1. Número de pacientes correspondientes a cada nivel neurológico de la lesión:(I) en o por encima de L2; (II) L3-L5; (III) en o por debajo de SI
3) La lesión pudenda superior viene dada por la existencia de disinergia vesicoesfinteriana, o por la ausencia del control voluntario del esfínter anal, manteniendo la sensibilidad perineal, el tono anal y el reflejo bulbocavernoso. En la lesión pudenda inferior, están alterados la sensibilidad perineal, el reflejo bulbocavernoso y el tono y control voluntario anal.

A su vez, según los resultados del diagnóstico de la lesión de los distintos componentes de la inervación vesicouretral, hemos clasificado la disfunción neurógena del tracto urinario inferior de nuestros pacientes, en base al nivel de la lesión y al tipo de inervación lesionada, de forma que:

1) Según el nivel de la lesión, se establecen dos categorías: Lesión inferior, que incluye la lesión de los componentes periféricos, medulares y de las vías de conexión periférico-medulares, y Lesión superior, que incluye la lesión de los componentes encefálicos y de las vías de conexión encéfalo-medulares.

2) Según el tipo de inervación lesionada, podemos distinguir: Lesiones únicas, cuando se afecta exclusivamente un solo elemento de la inervación vesicouretral (pudendo, simpático o parasimpático), pudiendo ser puras, si se afecta un solo nivel, o mixtas, si se afectan ambos niveles: superior e inferior. Lesiones múltiples, en las que existe afectación de más de un elemento de la inervación vesicouretral, y que a su vez también pueden ser puras o mixtas. Las puras, según el nivel de afectación se dividen en lesiones de tipo neurona motora inferior y de tipo neurona motora superior.

Los resultados estadísticos se obtuvieron, tras la inclusión de los datos de los pacientes en una base informática para su proceso mediante el programa SPSS, mediante la realización de la prueba de la chi cuadrado para comparación de proporciones, considerándose significativos valores de $p<0,05$.

\section{RESULTADOS}

En la exploración física, se pudo objetivar un nivel neurológico de la lesión, en o por encima de $\mathrm{L}_{2}$ (Nivel I) en 11 pacientes (21.1\%). Presentaban un nivel neurológico entre $L_{3}-L_{5}$ (Nivel II) 20 pacientes $(38.4 \%)$, mientras que otros $21(40.3 \%)$ tenían su lesión en o por debajo de S1 (Nivel III) (Figura 1). No existieron diferencias significativas en el nivel neurológico por sexos $(p=0,26)$.

La sensibilidad perineal fue normal en 10 casos $(24.3 \%)$, estaba disminuida en $21(57.5 \%)$, y 10 pacientes $(25 \%)$ la tenían totalmente abolida. Tenían un tono anal normal 11 pacientes $(26.8 \%)$, mientras 
que en los restantes $30(73.1 \%)$ este se encontraba disminuido. Lograban ejercer un adecuado control voluntario sobre el esfínter anal 4 pacientes 19.75 $\%)$, mientras que en 3 casos $(7.3 \%)$ se apreció un control disminuido, y en 34 (82.9\%) no existía dicho control. En los 35 pacientes en los que exploramos el reflejo bulbocavernoso, apreciamos que este se encontraba presente en 8 casos $(22.8 \%)$ y la ausencia del mismo en los 27 restantes $(77.1 \%)$.

La capacidad vesical se encontraba disminuida en 16 pacientes $(30,7 \%)$, pudiéndose considerar normal en los otros $36(69.2 \%)$, encontrándose una relación significativa entre su disminución y la presencia de incontinencia durante el estudio $(p=0,01)$. Presentaban residuo postmiccional 42 pacientes $(80.7 \%)$.

En cuanto al comportamiento del detrusor, en la gran mayoría de los pacientes se objetivo arreflexia vesical durante la exploración urodinámica $(88.4 \%)$. A esta arreflexia se asociaba una acomodación vesical disminuida en 22 pacientes $(42.3 \%)$, mientras que otros $24(46.1 \%)$ mantenían una acomodación normal. En 3 casos (5.7\%) objetivamos hiperreflexia del detrusor durante el llenado, y en otros 3 no se apreciaron alteraciones significativas en el comportamiento del detrusor tanto en su fase de llenado como en la de vaciado (Figura 2).

Comprobamos la incompetencia del sistema esfinteriano durante el llenado en 27 pacientes (51.9\%). En un caso se había realizado una cervicotomía vesical endoscópica durante la infancia y 4 pacientes tenían su cuello vesical desestructurado tras la retirada de una prótesis de incontinencia. En 22 pacientes $(42.3 \%)$ el sistema esfinteriano se catalogó

\section{COMPORTAMIENTO DEL DETRUSOR}

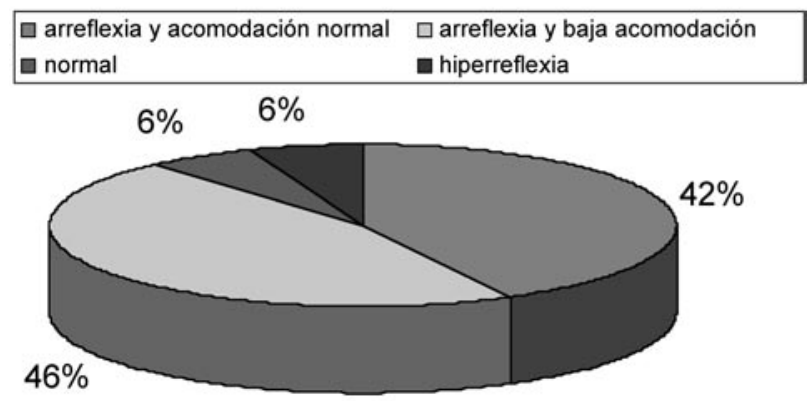

FIGURA 2. Comportamiento del detrusor en 52 adultos con mielomeningocele. como competente durante el llenado, puesto que aunque permanecían fijos, sin cambios en su actividad durante el mismo, generaban presiones suficientes para mantener la continencia. De estos 22 pacientes, $7(13.4 \%)$ debían su competencia esfinteriana a la presencia de un esfínter artificial previamente implantado, en 3 de ellos no funcionante. En $3(5.7 \%)$ de estos 22 pacientes, se había realizado una ampliación vesical (en dos de ellos junto al implante de la prótesis de incontinencia y en el otro como medida aislada) que favorecían la presencia de competencia esfinteriana. Por último, el sistema esfinteriano tuvo un comportamiento normal durante el llenado, en 3 pacientes (5.7\%) (Figura 3 ).

Durante la fase de vaciado vesical, en la mayoría de los casos, comprobamos la ausencia de relajación del sistema esfinteriano. Este comportamiento lo presentaron 43 pacientes $(82.6 \%)$, debiéndose en $3(5.7 \%)$ de ellos a la presencia de restos afuncionantes de prótesis de incontinencia. En 2 casos (38\%) pudimos detectar la presencia de un comportamiento disinérgico del sistema esfinteriano distal, durante las contracciones detrusorianas de hiperreflexia que presentaban. En los restantes 7 pacientes (13.4\%), consideramos que su sistema esfinteriano tenía un comportamiento sinérgico, $3(5.7 \%)$ de ellos gracias al comportamiento normal del mismo y los otros 4 $(7.6 \%)$ debido a la presencia de un esfínter artificial funcionante (Figura 4).

Además de los hallazgos de imagen del sistema esfinteriano que contribuyeron a catalogar el comportamiento del mismo, durante el estudio videourodinámico pudimos comprobar la presencia de un contorno anormal del cistograma en 37 pacientes $(71.1 \%)$, variable desde discretas lobulaciones a

\section{SISTEMA ESFINTERIANO DURANTE EL LLENADO}
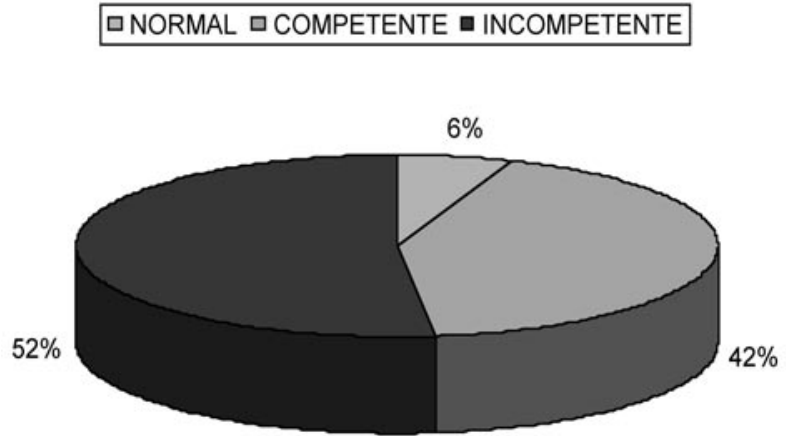

FIGURA 3. Comportamiento del sistema esfinteriano durante el llenado. 
SISTEMA ESFINTERIANO DURANTE EL VACIADO

\section{SIN RELAJACIÓN $\square$ DISINÉRGICO ■ SINÉRGICO}

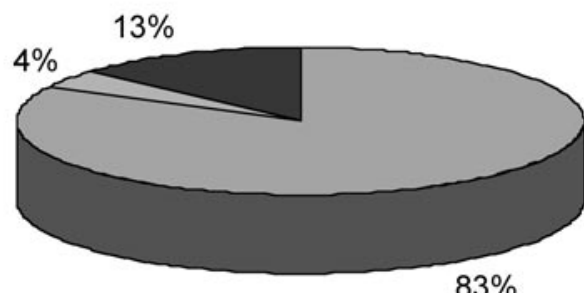

FIGURA 4. Comportamiento del sistema esfinteriano durante el vaciado.

una morfología microdiverticular y sin relación con el nivel neurológico de la lesión ( $p=0.68)$. Igualmente descubrimos la presencia de reflujo vesicoureteral en 8 pacientes (15.3\%). En 6 casos (11.5\%) el reflujo era unilateral, y en dos de estos, refluía a remanentes ureterales dejados tras una nefrectomía. En los otros 2 casos (3.8\%) el reflujo fue bilateral, en uno de ellos refluyendo, también, a un segmento ureteral remanente postnefrectomía.

La existencia de reflujo no se relacionó con el nivel neurológico de la lesión $(p=0,24)$, ni con el comportamiento del sistema esfinteriano, tanto durante el llenado $(p=0,90)$, como durante el vaciado $(p=0.26)$, encontrándosele una relación estadísticamente significativa con la capacidad vesical disminuida $(p=0,004)$ y con la presencia de arreflexia con acomodación vesical disminuida $(p=0,03)$.

Diagnóstico de la lesión simpática: En 21 casos $(40.3 \%)$, se evidenció lesión simpática inferior, siendo 3 de ellos de tipo I, 12 de tipo II, y los 6 restantes de tipo III. No encontramos en ningún paciente datos de lesión simpática superior. En 17 (32.6\%) pacientes no se evidenció lesión simpática, y en otros $14(26.9 \%)$ esta no se pudo determinar por la existencia de antecedentes quirúrgicos que impedían una valoración correcta (Figura 5).

Diagnóstico de la lesión parasimpática: Solamente apreciamos lesión parasimpática superior en 3 casos $(5.7 \%)$, mientras que en $43(82.6 \%)$ objetivamos lesión parasimpática inferior. Tres pacientes no tenían lesión parasimpática y en otros 3 esta no era valorable al habérseles realizado una ampliación vesical (Figura 6).

Diagnóstico de la lesión pudenda: Encontramos 6 casos de lesión pudenda superior (11.5\%);

\section{LESIÓN SIMPÁTICA}

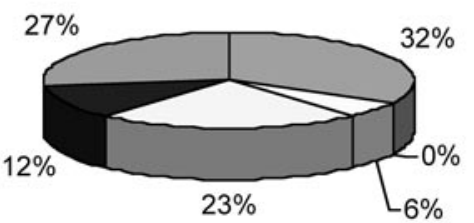

$\square$ SIN LESIÓN

๑ SUPERIOR

口 INFERIOR TIPO I $\square$ INFERIOR TIPO II — INFERIOR TIPO III NO VALORABLE

FIGURA 5. Presencia de lesión simpática y tipo de ésta en 52 adultos con mielomeningocele.

otros 33 pacientes, se catalogaron como afectos de lesión pudenda inferior (63.4\%). Cuatro pacientes no presentaban datos de lesión pudenda $(7.6 \%)$ y en 9 pacientes (17.3\%) ésta no se pudo determinar de forma concluyente (Figura 7).

Tipos de disfunción neurógena del tracto urinario inferior: De los 52 pacientes objeto del estudio, en 3 de ellos (5.7\%) no se encontraron alteraciones en ninguno de los tres componentes de su inervación vesicouretral, siendo sus exploraciones neurourológicas y urodinámicas normales. De los 49 pacientes, en los que si se detectaron alteraciones, pudimos realizar una adecuada determinación de los tres tipos de inervación en 29 pacientes, resultando que salvo en un solo caso, en el que existía una lesión única, en concreto del parasimpático inferior, todos los demás presentaban lesiones múltiples, al verse afectado más de un tipo de inervación.

\section{LESIÓN PARASIMPÁTICA}

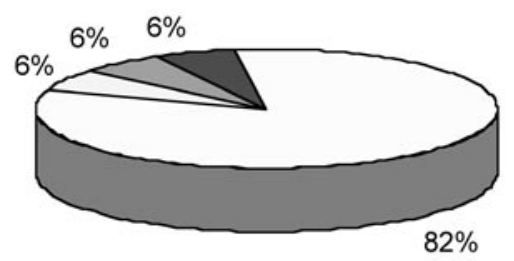

$\square$ SIN LESION $\square$ SUPERIOR $\square$ INFERIOR $\square$ NO VALORABLE

FIGURA 6. Lesión parasimpática en los 52 adultos con mielomeningocele. 


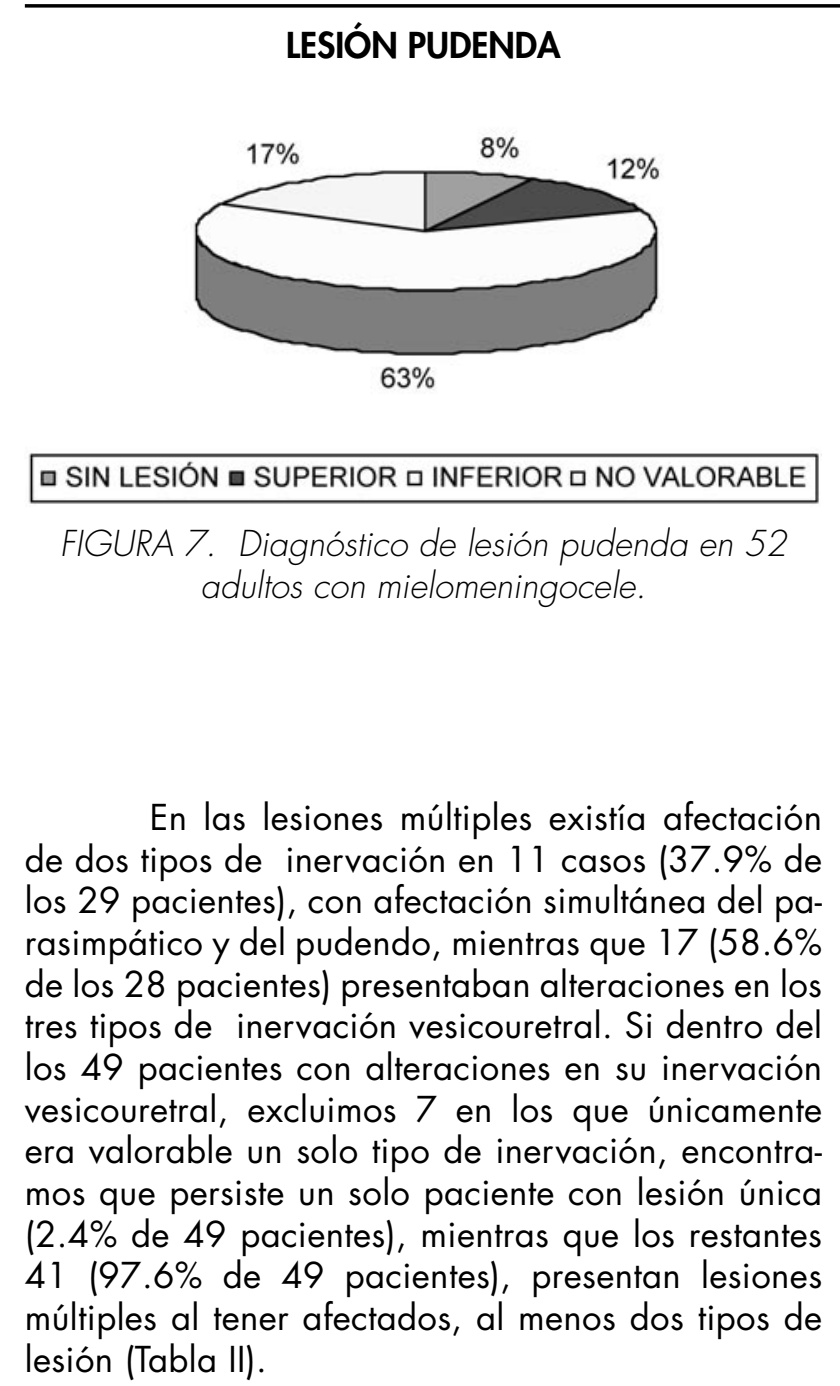

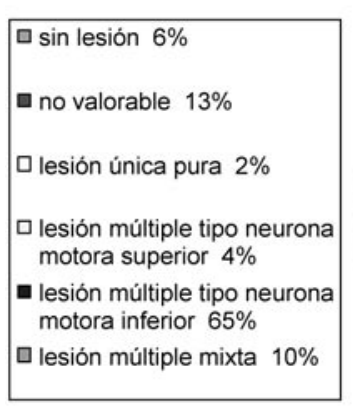

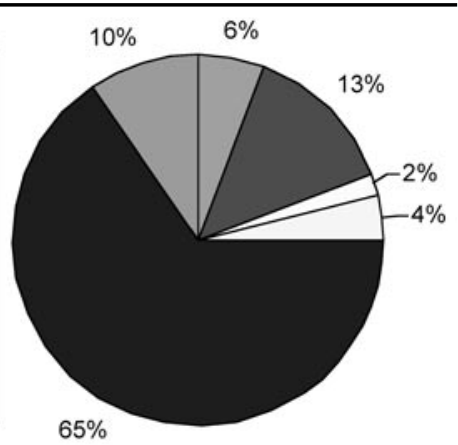

FIGURA 8. Tipos de disfunción neurógena del tracto urinario inferior en 52 adultos nacidos con mielomeningocele.

De estos 41 pacientes con lesiones múltiples, se demostró lesión múltiple pura tipo neurona motora superior en 2 casos (3.8\%), lesión múltiple pura tipo neurona motora inferior en $34(65.3 \%)$ y lesiones múltiples mixtas en 5 casos $(9.6 \%)$. Las lesiones múltiples mixtas, correspondieron a 2 casos de lesión parasimpática superior junto a lesión pudenda inferior, sin alteración de la vía simpática, otros 2 casos de lesión parasimpática inferior y pudenda superior, asociadas a lesión simpática inferior tipo Il, y un último caso de lesión parasimpática inferior con pudenda superior y simpática inferior tipo III. No encontramos una relación estadísticamente significativa entre estos tipos de disfunción neurógena y el nivel neurológico de la lesión (Figura 8).

\section{TABLA II. TIPO DE LESIÓN SEGÚN EL NÚMERO DE ELEMENTOS DE LA INERVACIÓN VESICOURETAL AFECTADOS.}

En el grupo de 29 pacientes, en los que se pudo explorar los tres tipos de inervación vesicouretral

Lesiones únicas:

lesión parasimpática.

Lesiones múltiples:

lesión parasimpática y pudenda. $37.9 \%$

lesión simpática, parasimpática y pudenda. $.58 .6 \%$

En 42 pacientes en los que se pudieron explorar al menos dos, de los tres tipos de inervación vesicouretral

Lesiones únicas. $.2 .3 \%$

Lesiones múltiples. $97.6 \%$ 


\section{DISCUSIÓN}

La esperanza de vida de los nacidos con MMC, ha ido aumentando de una manera espectacular en los últimos cincuenta años. Condenados durante siglos a una muerte temprana, como consecuencia de las graves alteraciones secundarias a la malformación y en especial la frecuente hidrocefalia que la acompaña, no fue hasta mediados del siglo $X X$, gracias a diversos avances médicos y fundamentalmente al desarrollo de las válvulas de derivación ventricular, cuando estos pacientes comenzaron a tener ciertas expectativas de supervivencia.

Pero pronto se comprobó que este aumento en la supervivencia no se acompañaba de una mejoría en las graves discapacidades que los enfermos presentaban, por lo que se planteó si iniciar el tratamiento de estos niños era algo cruel y por tanto no era mejor dejarlos morir. Con esta idea, a principios de la década de los setenta, se establecieron criterios de selección orientados a discriminar los pacientes que no debían ser tratados (12).

Estudios posteriores, cotejando resultados entre series de niños en las que se habían aplicado o no, estos criterios de selección, demostraron que ninguno de ellos, ni aislado ni en combinación, tenían valor pronóstico, concluyendo que al menos el $60 \%$ de los niños a los que se había dejado morir, podrían haber tenido una vida útil si se les hubiera dado la oportunidad $(13,14)$.

Posteriores avances médicos, entre los que destacan el perfeccionamiento de los estudios urodinámicos y la generalización de los cateterismos intermitentes, han permitido una supervivencia en este grupo de pacientes jamás alcanzada, de forma que al inicio del presente siglo se espera que casi el $75 \%$ de los nacidos con MMC alcancen la mayoría de edad (15), cifrándose la probabilidad de supervivencia a los 30 años en el $49 \%$ (16).

Aunque la exploración física neurourológica no presenta, en el adulto con MMC dificultades especiales para su realización, la de los estudios urodinámicos se ve dificultada por diversos factores. Así determinadas deformidades óseas y contracturas musculares, presentes en algunos pacientes, dificultan la colocación de electrodos para el estudio de la musculatura perineal. Igualmente, la reproductibilidad de su incontinencia de esfuerzo, se ve dificultada por la poca capacidad de prensa abdominal presente en algunos casos, que hace que no pueda objetivarse con la tos en el decúbito, cuando ésta es patente al pasar a la posición de bipedestación. Además, la especial configuración esquelética de algunos afectos, sobre todo en caso de cifoescoliosis pronunciadas, puede ocasionar una superposición anormal de la imagen radiológica del aparato urinario, sobre estructuras óseas o incluso sobre sí mismo, de forma que dificulte e incluso impida una correcta visualización de un reflujo vesicoureteral.

Estos pacientes presentan una multiplicidad de lesiones en su disfunción neurourológica, con predominio de la lesión múltiple pura tipo motoneurona inferior, y al igual que ocurre durante la infancia (17), no hemos encontrado una relación entre el tipo de disfunción vesicouretral que presentan y el nivel neurológico de su lesión. Por tanto, creemos que a los dos mayores determinantes de la incapacidad y dependencia de estos pacientes, la extensión del déficit neurológico y el coeficiente intelectual (18), se debería añadir el estado funcional de su aparato urinario.

Encontramos un gran predominio de la arreflexia vesical, presente en 43 de los 49 pacientes $(87.7 \%)$, en los que se pudo evaluar dicha función (los 3 restantes tenían una ampliación vesical) que contrasta con los datos obtenidos en los estudios urodinámicos realizados en los primeros meses de vida de este tipo de pacientes.

El porcentaje de arreflexia vesical, en el período neonatal, se cifra alrededor del $43 \%$ (19), porcentaje que no se ha modificado en una reciente serie de niños, en los que se ha realizado el cierre de su lesión durante la gestación (20), y que puede incluso disminuir en los primeros meses y años de la vida (21). Pero su valor aumenta cuando se analizan series con niños de mayor edad, en las que el porcentaje de arreflexia vesical oscila entre el 53 y $83 \%(7,22,23)$, por lo que la edad de los pacientes parece influir en los resultados del comportamiento del detrusor.

En el paciente con $M M C$, cuando la vejiga mantiene su capacidad de contraerse, el cuello vesical puede ser competente $o$ incompetente, pero nunca es obstructivo, y el mecanismo esfinteriano distal, es casi siempre dinámicamente obstructivo, por la existencia de disinergia vesicoesfinteriana. Cuando la capacidad contráctil vesical esta disminuida o ausente, el cuello suele ser incompetente, y el mecanismo esfinteriano distal siempre es, en mayor o menor medida, tanto estáticamente obstructivo como incompetente con los esfuerzos.

Este ha sido precisamente, el comportamiento más habitual del sistema esfinteriano en nuestros pacientes, al permanecer el mismo fijo, apreciándose una ausencia de relajación durante los intentos 
de vaciado vesical (83\%). Esta situación funcional, puede deberse tanto a la persistencia, más o menos completa, de la inervación pudenda sacra, como a la existencia de un esfínter denervado con un componente fibrótico añadido.

En un pequeño número de recién nacidos con $M M C$, no se encuentran alteraciones significativas en sus estudios urodinámicos, si bien el porcentaje inicial puede descender fundamentalmente durante los 6 primeros años de vida, como consecuencia de su médula anclada (24), estableciéndome entre el $4.6-8.6 \%(25,26)$, cifras que concuerdan con la del $5.7 \%$ obtenida en nuestros pacientes adultos.

Los escasos estudios urológicos realizados en adultos con $M M C$, se centran más en la situación clínica de estos pacientes que en los hallazgos urodinámicos.

En un trabajo en el que se constatan estos, realizado en 33 pacientes mayores de 16 años (27), se identificó una actividad contráctil normal del detrusor en 3 casos $(9 \%)$, mientras que 5 presentaban hiperreflexia $(15 \%)$ y 25 se catalogaron como hiporrefléxicos $(75.7 \%)$, resultados similares a los obtenidos por nosotros.

Los pacientes con esta malformación se han clasificado en grupos de alto y de bajo riesgo de daño renal, según la presión vesical. La hiperpresión vesical, a causa de una disinergia vesicoesfinteriana o por una mala acomodación vesical con un sistema esfinteriano competente, se ha identificado como el mayor factor de riesgo renal $(7,28)$, por lo que se ha insistido en la necesidad de su detección y tratamiento precoz (29).

Pese a esto, el fracaso renal, junto a problemas funcionales de la válvula de derivación, continúan siendo los principales responsables de la persistencia del riesgo del $1 \%$ de muerte anual, presente desde los 5 a los 30 años en estos pacientes (30).

Por tanto, el hecho de una menor supervivencia en pacientes con determinadas disfunciones o la corrección quirúrgica de estas, contribuiría a el predominio de situaciones de bajo riesgo renal en adultos con MMC, como ocurrió en nuestros pacientes, si bien las diversas complicaciones que hemos observado en el seguimiento de los mismos (31), junto a la necesidad de controlar disfunciones de riesgo renal, justifican el seguimiento periódico de todos ellos.

\section{CONCLUSIONES}

1.- Los pacientes adultos nacidos con MMC pueden presentar distintos tipos de disfunción vesicouretral, como consecuencia de la persistencia de su lesión neurológica.

2.- El tipo de disfunción vesicouretral en estos pacientes, es independiente del nivel neurológico de su lesión.

3.- Existe un claro predominio del comportamiento arrefléxico del detrusor y de la ausencia de relajación del sistema esfinteriano durante los intentos de vaciado vesical.

4.- La disfunción neurógena del tracto urinario inferior más frecuente en estos pacientes es la lesión múltiple pura tipo neurona motora inferior.

5.- En un pequeño porcentaje de pacientes persiste la ausencia de alteraciones demostrables en sus estudios urodinámicos.

6.- La presencia de la disfunción vesicouretral, algunas de ellas asociadas a riesgo de deterioro del aparato urinario superior, justifica el seguimiento urológico de por vida de estos pacientes.

\section{BIBLIOGRAFÍA y LECTURAS RECOMENDADAS ( ${ }^{*}$ lectura de interés $y^{* *}$ lectura fundamental)}

1. BAUER, S.B.; LABICK, K.B.; DIEPPA, R.A. y cols.: "Urodynamic evaluation in a boy with myelodysplasia and incontinence". Urology, 10: 354, 1977.

**2. BAUER, S.B.: "Neurogenic dysfunction of the lower urinary tract in children". En Walsh P.C., Retik A.B., Vaughan E.D., Wein A.J.: Campbell's Urology / Ed Vol 2, Saunders, Philadelphia, 65: 2019, 1998.

3. WEBSTER, G.D.; EL-MAHROUKY, A.; STONE, A.R. y cols.: "The urological evaluation and management of patients with myelodysplasia". BJU, 58: 261, 1986.

4. LAIS, A.; KASABIAN, N.G.; DYRO, F.M. y cols.: "Neurosurgical implications of continuous neuro-urological surveillance of children with myelodysplasia". J. Urol., 150: 1879, 1993.

5. BEGGER, J.H.; MEIHOIZEN DE REGT, M.J.; HOGEN ESCH, I. y cols.: "Progressive neurologic deficit in children with spina bifida aperta". Z. Kinderchir, 41: 13, 1986. 
*6. SPINDEL, M.R.; BAUER, S.B.; DYRO, F.M. y cols.: "The changing neuro-urologic lesion in myelodysplasia". JAMA, 258: 1630, 1987.

**7. McGUIRE, E.J.; WOODSIDE, J.R.; BORDEN, T.A. y cols.: "The prognosis value of urodynamic testing in myelodysplastic patients". J. Urol., 126: 205, 1981.

*8. SIDI, A.A.; DYKSTRA, D.D.; GONZÁLEZ, R.: "The value of urodynamic testing in the management of neonates with myelodysplasia: A prospective study". J. Urol., 135: 90, 1986.

9. SHURTLEFF, D.B.; HAYDEN, P.W.; CHAPMAN, W.H. y cols.: "Myelodysplasia. Problems of long-term survival and social function". West J. Med., 122: 199, 1975.

10. ABRAMS, P.; CARDOZO, L.; FASLL, M. y cols.: "The standardisation of terminology of lower urinary tract funtion: Report from the standardisation sub-comittee of International Continence Society". Neurourol Urodyn. 21:167, 2002.

11. VIRSEDA, M.; SALINAS, J.; ADOT, J.M. y cols.: "Guías urodinámicas V. Urodinámica en neurourología". Urod. A., 14: 59, 2001.

12. LORBER, J.: "Results of treatment of myelomeningocele. An analysis of 524 unselected cases, with special reference to possible selection for treatment". Dev. Med. Child Neurol., 13: 279, 1971.

13. McLONE, D.G.: "Results of treatment of children born with a myelomeningocele". Clin. Neurosurg., 30: 407, 1983.

*14. McLONE, D.G.: "Treatment of myelomeningocele: Arguments against selection". En Little J R : Clinical Neurosurgery. Williams and Wilkins. Baltimore, 33: 359, 1986.

15. BOWMAN, R.M.; McLONE, D.G.; GRANT, J.A. y cols.: "Spina Bifida Outcome: A 25 Year Prospective". Pediatr. Neurosurg., 34: 114, 2001.

16. HUNT, G.M.; PALMER, C.: "The medial survival time in open spina bifida". Dev. Med. Child Neurol., 39: 568; 1997.

17. WYNDAELE, J.J.; DE SY, W.A.: "Correlation between the findings of a clinical neurological examinations and the urodynamic dysfunction in children with myelodysplasia”. J. Urol., 133: 638, 1985.

18. HUNT, G.M.; POULTON, A.: "Open spina bifida: A complete cohort reviewed 25 years after closure". Dev. Med. Child Neurol., 37: 19, 1995.

19. BAUER, S.B.: "Myelodysplasia: Newborn evaluation and management". En McLaurin R.L. Ed: Spina bifida: A Multidisciplinary Approach. NewYork, Praeger, 262-267, 1984.
20. HOLZBEIERLEIM, J.; POPE, J.C.; ADAMS, M.C. y cols.: "The urodynamic profile of myelodysplasia in childhood with spinal closure during gestation”. J. Urol., 164: 1336, 2000.

21. SILLEN, U.; HANSSON, E.; HERMANSSON, G. y cols.: "Development of the urodynamic pattern in infants with mielomeningocele". Br. J. Urol., 78: 596, 1996.

22. AGÜERA, L.; SÁNCHEZ, P.; ROSELL, D. y cols.: "Análisis de los factores urodinámicos determinantes de la aparición de hidronefrosis y daño renal en pacientes con mielodisplasia". Urod. A., 3: 193, 1991.

23. SAKAKIBARA, R.; HATTORI, T.; UCHIYAMA, Y. y cols.: "Uroneurological assessment of spina bifida cystica and occulta". Neurourol. Urodyn., 22: 328, 2003.

24. TARCAN, T.; BAUER, S.B.; OLMEDO, E. y cols.: "Long-tern followup of newborns with myelodysplasia and normal urodynamics findings: Is followup necessary?". J. Urol., 165: 564, 2001.

25. REINER, I.; JONES, M.; DONNELL, S. y cols.: "Incidence of normal micturition in myelomeningocele patients". Arch. Dis. Child, 67: 640, 1992.

**26. LIE, H.R.; LAGERGREN, J.; RASMUSSEN, F. y cols.: "Bowel and bladder control of children with myelomeningocele: Nordic study". Dev. Med. Child Neurol., 33: 1053, 1991.

27. GLOTT, T.; STANGHELLE, J.K.; RAND-HENDRIKSEN, S. y cols.: "Voiding problems and urinary tract complications in adolescent and adults with mielomeningocele". Tidsskr Nor Laegeforen, 121: 1211, 2001.

*28. GHONIEM, G.M.; ROACH, M.B.; LEWIS, V.H. y cols.: "The value of leak pressure and bladder compliance in the urodynamic evaluation of meningomyelocele patients". J. Urol., 144: 1140, 1990.

29. ROMERO, J.; PERALES, L.; RODRÍGUEZ, J.M. y cols.: "Destino renal en los pacientes mielodisplásicos con disfunciones vesicoesfinterianas de riesgo no tratadas". Actas Urol. Esp., 13: 357, 1989.

30. HUNT, G.M.: "Non-selective intervention in newborn babies with open spina bifida: The outcome 30 years on for the complete cohort". Eur. J. Pediatr. Surg., 9: 5, 1999.

31. VERDÚ TARTAJO, F.: "Caracterización neurourológica del mielomeningocele en el paciente adulto". Tesis Doctoral. Facultad de Medicina. Universidad Complutense de Madrid. 2005. 